Pacific

Journal of

Mathematics

\title{
VOLUME ENTROPY OF HILBERT GEOMETRIES
}

Gautier Berck, Andreas Bernig and Constantin Vernicos 


\title{
VOLUME ENTROPY OF HILBERT GEOMETRIES
}

\author{
Gautier Berck, Andreas Bernig ANd Constantin Vernicos
}

\begin{abstract}
We show that among all plane Hilbert geometries, the hyperbolic plane has maximal volume entropy. More precisely, we show that the volume entropy is bounded above by $2 /(3-d) \leq 1$, where $d$ is the Minkowski dimension of the extremal set of $K$, and we construct an explicit example of a plane Hilbert geometry with noninteger volume entropy. In arbitrary dimension, the hyperbolic space has maximal entropy among all Hilbert geometries satisfying some additional technical hypothesis. To achieve this result, we construct a new projective invariant of convex bodies, similar to the centroaffine area.
\end{abstract}

\section{Introduction}

In his famous Fourth Problem, Hilbert asked for a characterization of metric geometries whose geodesics are straight lines. He constructed a special class of examples, now called Hilbert geometries [Hilbert 1895; 1999], which have since attracted much interest; see, for example, [Nasu 1961; de la Harpe 1993; Karlsson and Noskov 2002; Socié-Méthou 2004; Foertsch and Karlsson 2005; Benoist 2006; Colbois and Vernicos 2007], and the two complementary surveys [Benoist 2008] and [Vernicos 2005].

A Hilbert geometry is a particularly simple metric space on the interior of a compact convex set $K$ (see the definition below). This metric happens to be a complete Finsler metric whose set of geodesics contains the straight lines. Since the definition of the Hilbert geometry only uses cross-ratios, the Hilbert metric is a projective invariant. In the particular case where $K$ is an ellipsoid, the Hilbert geometry is isometric to the usual hyperbolic space.

An important part of the above mentioned works, and of older ones, is to study how different or close to the hyperbolic geometry these geometries can be. For instance, if $K$ is not an ellipsoid, Kay [1967, Corollary 1] showed that the metric is never Riemannian. This result is related to the fact that among all finitedimensional normed vector spaces, many notions of curvatures are only satisfied

MSC2000: 51F99, 53A20, 53C60.

Keywords: metric geometry, Hilbert geometry, convex geometry.

Berck and Bernig were supported by the Schweizerischer Nationalfonds grants SNF PP002-114715/1 and 200020-121506/1. 
by the Euclidean spaces [Kelly and Paige 1952; Kelly and Straus 1958; 1968]. However, if $\partial K$ is sufficiently smooth, then the flag curvature, an analog of the sectional curvature, of the Hilbert metric is constant and equals -1; see [Shen 2001, Example 9.2.2]. Hence one can ask whether or not these geometries behave like negatively curved Riemannian manifolds. The example of the triangle geometry that is isometric to a two-dimensional normed vector space shows that things are a little more involved (see [de la Harpe 1993], and also theorems cited below). The present work is partially inspired by the feeling that Hilbert geometries might be thought of as geometries with Ricci curvature bounded from below, and focuses on the volume growth of balls.

Unlike the Riemannian case, where there is only one natural choice of volume, there are several good choices of volume on a Finsler manifold. We postpone this issue to Section 2 and fix just one volume (like the $n$-dimensional Hausdorff measure) for the moment.

Let $B(o, r)$ be the metric ball of radius $r$ centered at $o$. The volume entropy of $K$ is defined by the limit (provided it exists)

$$
\text { Ent } K:=\lim _{r \rightarrow \infty} \frac{\log \operatorname{Vol} B(o, r)}{r} \text {. }
$$

The entropy depends neither on the particular choice of the base point $o \in \operatorname{int} K$, nor on the particular choice of the volume. If $h=$ Ent $K$, then $\operatorname{Vol} B(o, r)$ behaves roughly as $e^{h r}$.

It is well known and easy to prove (see S. Gallot, D. Hulin and J. Lafontaine [Gallot et al. 2004, Section III.H]) that the volume of a ball of radius $r$ in the $n$-dimensional hyperbolic space is given by

$$
n \omega_{n} \int_{0}^{r}(\sinh s)^{n-1} d s=O\left(e^{(n-1) r}\right),
$$

where $\omega_{n}$ is the volume of the Euclidean unit ball of dimension $n$. It follows that the entropy of an ellipsoid equals $n-1$.

In general, it is not known whether the limit above exists, although it does in several cases: It exists if the convex set $K$ is divisible, which means that a discrete subgroup of the group of isometries of the Hilbert geometry acts cocompactly [Benoist 2004]. If the convex set is sufficiently smooth (for example, $C^{2}$ with positive curvature suffices), the entropy exists and equals $n-1$ (see the theorem of Colbois and Verovic below). In general, one may define lower and upper entropies Ent and $\overline{\text { Ent }}$ by replacing the limit in the definition (1) by lim inf or lim sup.

There is a well known conjecture (whose origin seems difficult to locate) saying that the hyperbolic space has maximal entropy among all Hilbert geometries of the same dimension: 
Conjecture. For any n-dimensional Hilbert geometry, $\overline{\text { Ent }} K \leq n-1$.

Notice that an analogous result in Riemannian geometry is a consequence of Bishop's volume comparison theorem for complete Riemannian manifolds of Ricci curvature bounded by $-(n-1)$ [Gallot et al. 2004, Theorem 3.101(i)].

Several particular cases of the conjecture were treated in the literature. The following one shows that the volume entropy does not characterize the hyperbolic geometry among all Hilbert geometries.

Theorem [Colbois and Verovic 2004]. If $K$ is $C^{2}$-smooth with strictly positive curvature, then the Hilbert metric of $K$ is bi-Lipschitz to the hyperbolic metric and therefore Ent $K=n-1$.

The case of convex polytopes is rather well understood.

Theorem [Bernig 2009; Vernicos 2008b]. The Hilbert metric associated to a convex body $K$ is bi-Lipschitz to a normed space if and only if $K$ is a polytope. In particular, the entropy of a polytope is 0.

The two-dimensional case was earlier obtained by Colbois, Vernicos, and Verovic in [Colbois et al. 2008].

Instead of taking the volume of balls, a natural choice is to study the volume growth of the metric spheres $S(o, r)$. One may define a (spherical) entropy by

$$
\mathrm{Ent}^{s} K:=\lim _{r \rightarrow \infty} \frac{\log \operatorname{Vol} S(o, r)}{r},
$$

provided the limit exists. In general, one may define upper and lower spherical entropies $\overline{\mathrm{Ent}}^{s} K$ and $\underline{\mathrm{Ent}}^{s} K$ by replacing the limits in (2) by a lim sup or lim inf.

The next theorem is a spherical version of the theorem of Colbois and Verovic.

Theorem [Borisenko and Olin 2008]. If $K$ is an n-dimensional convex body of class $C^{3}$ with positive Gauss curvature, then $\mathrm{Ent}^{s}=n-1$.

Our first main theorem treats the two-dimensional case. Recall that an extremal point of a convex body $K$ is a point that is not a convex combination of two other points of $K$.

First main theorem. Let $K$ be a two-dimensional convex body. Let $d$ be the upper Minkowski dimension of the set of extremal points of $K$. Then the entropy of $K$ is bounded by

$$
\overline{\operatorname{Ent}} K \leq \frac{2}{3-d} \leq 1
$$

The inequality is sharp if $K$ is smooth or contains some positively curved smooth part in the boundary. In this case the upper Minkowski dimension of ex $K$ and the entropy are both 1 . On the other hand, for polygons the upper Minkowski 
dimension of the set of extremal points and the entropy both vanish (see the theorem of [Colbois et al. 2008]), and the inequality is not sharp in this case.

It should be noted that the entropy behaves in a rather subtle way (see also [Vernicos 2008a] for a technical study of the entropy, complementary to this paper). As we have seen above, the entropy of a polygon vanishes. In contrast to this, we will construct a convex body with piecewise affine boundary whose entropy is between $1 / 4$ and $3 / 4$.

Our second main theorem applies in all dimensions. It weakens in a substantial way the assumptions in the theorem of Colbois and Verovic and strengthens its conclusions, for it gives not only the precise value of the entropy but also the entropy coefficient. To state it, we introduce a projective invariant of convex bodies, which is interesting in itself.

Let $V$ be an $n$-dimensional vector space with origin $o$. Given a convex body $K$ containing $o$ in the interior, we define a positive function $a$ on the boundary by the condition that for $p \in \partial K$ we have $-a(p) p \in \partial K$. The letter $a$ stands for antipodal. If $V$ is endowed with a Euclidean scalar product, we let $k(p)$ be the Gauss curvature and $n(p)$ be the outer normal vector at a boundary point $p$ (whenever they are welldefined, which is almost everywhere the case following [Alexandroff 1939]).

Definition. The centroprojective area of $K$ is

$$
\mathscr{A}_{p}(K):=\int_{\partial K} \frac{\sqrt{k}}{\langle n, p\rangle^{(n-1) / 2}}\left(\frac{2 a}{1+a}\right)^{(n-1) / 2} d A .
$$

It is not quite obvious (but true, as we shall see) that this definition does not depend on the choice of the scalar product. In fact, the centroprojective area is invariant under projective transformations fixing the origin. The reader familiar with the theory of valuations may notice the similarity with the centroaffine surface area, whose definition is the same except that the second factor (containing the function $a$ ) does not appear. We refer to [Laugwitz 1965; Leichtweiß 1998] for more information on affine and centroaffine differential geometry.

Second main theorem. If $\partial K$ is $C^{1,1}$ or if $n=2$, then

$$
\lim _{r \rightarrow \infty} \frac{\operatorname{Vol} B(o, r)}{\sinh ^{n-1} r}=\frac{1}{n-1} \mathscr{A}_{p}(K) .
$$

In the first case, $\mathscr{A}_{p}(K) \neq 0$ and hence Ent $K=n-1$.

Our next theorem, together with the previous ones, shows that it suffices to assume $K$ to be merely of class $C^{1,1}$ in the theorem of Borisenko and Olin.

Theorem. For each convex body $K$,

$$
\underline{\text { Ent }}^{s} K=\underline{\text { Ent }} K \text { and } \overline{\operatorname{Ent}}^{s} K=\overline{\operatorname{Ent}} K .
$$


Plan of the paper. In the next section, we collect some well-known facts about convex bodies, Hilbert geometries and volumes on Finsler manifolds, and we prove a number of easy lemmas. Using some inequalities for volumes in normed spaces, we show that entropy and spherical entropy coincide for general convex bodies.

In Section 3, we use the lemmas to prove our main theorems. In Section 4, we give an intrinsic definition of the centroprojective surface area and study some of its properties. In particular, we show that it is upper semicontinuous with respect to Hausdorff topology.

\section{Preliminaries on convex bodies and Hilbert geometries}

2.1. Convex bodies. Let $V$ be a finite-dimensional real vector space. By a convex body, we mean a compact convex set $K \subset V$ with nonempty interior (note that this last condition is sometimes not required in the literature). Most of the time, the convex bodies will be assumed to contain the origin in their interiors. In such a case, we will as usual call the Minkowski functional the positive, homogeneous function of degree 1 whose level set at height 1 is the boundary $\partial K$. It is a convex function, which by Alexandroff's theorem admits a quadratic approximation almost everywhere [Alexandroff 1939; Evans and Gariepy 1992, page 242]. In the following, boundary points where Alexandroff's theorem applies will be called smooth. If we assume the vector space to be equipped with an inner product, the principal curvatures of the boundary and its Gauss curvature $k$ are well defined at every smooth point.

We will be concerned with generalizations and variations of Blaschke's rolling theorem, a proof of which may be found in [Leichtweiß 1993].

Theorem 2.1 [Blaschke 1956]. Let $K$ be a convex body in $\mathbb{R}^{n}$ whose boundary is $C^{2}$ with everywhere positive Gaussian curvature. Then there are two positive radii $R_{1}$ and $R_{2}$ such that for every boundary point $p$, there exists a ball of radius $R_{1}$ (respectively $R_{2}$ ) containing $p$ on its boundary and contained in $K$ (respectively containing $K$ ).

We first remark that for the "inner part" of Blaschke's result, the regularity of the boundary may be lowered. Recall that the boundary of a convex body is $C^{1,1}$ provided it is $C^{1}$ and the Gauss map is Lipschitz continuous. Roughly speaking, the second condition says that the curvature of the boundary remains bounded, even if it is only almost everywhere defined. The following proposition then gives a geometrical characterization of such bodies [Hörmander 2007, Proposition 2.4.3; Bangert 1999; Hug 1999b].

Proposition 2.2. The boundary of a convex body $K$ is $C^{1,1}$ if and only if there exists some $R>0$ such that $K$ is the union of balls with radius $R$. 
Without assumption on the boundary, there is still an integral version of Blaschke's rolling theorem.

Theorem 2.3 [Schütt and Werner 1990]. For a convex body $K$ containing the unit ball of a Euclidean space and $p \in \partial K$, let $R(p) \in[0, \infty)$ be the radius of the biggest ball contained in $K$ and containing $p$. Then for all $0<\alpha<1$,

$$
\int_{\partial K} R^{-\alpha} d \mathscr{H}^{n-1}<\infty .
$$

We will need the following refinement of this theorem.

Proposition 2.4. In the same situation as in Theorem 2.3, for each Borel subset $B \subset \partial K$ we have

$$
\int_{B} R^{-\alpha} d \mathscr{H}^{n-1} \leq 2(n-1)^{\alpha}\left(\frac{2^{\alpha}}{1-2^{\alpha-1}}\right)^{\alpha}\left(\mathscr{H}^{n-1}(B)\right)^{1-\alpha}\left(\mathscr{H}^{n-1}(\partial K)\right)^{\alpha} .
$$

In particular, for some constant $C$ depending on $K$, we have

$$
\int_{B} R^{-1 / 2} d \mathscr{H}^{n-1} \leq C\left(\mathscr{H}^{n-1}(B)\right)^{1 / 2} .
$$

Proof. By [Schütt and Werner 1990, Lemma 4], we have for $0 \leq t \leq 1$

$$
\mathscr{H}^{n-1}(\{p \in \partial K \mid R(p) \leq t\}) \leq(n-1) t \mathscr{H}^{n-1}(\partial K),
$$

from which we deduce that, for each $0<\epsilon<1$,

$$
\begin{aligned}
\int_{\partial K \cap\{R<\epsilon\}} R^{-\alpha} d \mathscr{H}^{n-1} & =\sum_{i=0}^{\infty} \int_{\partial K \cap\left\{\epsilon 2^{-i-1} \leq R<2^{-i} \epsilon\right\}} R^{-\alpha} d \mathscr{H}^{n-1} \\
& \leq \sum_{i=0}^{\infty}\left(\epsilon 2^{-i-1}\right)^{-\alpha} \mathscr{H}^{n-1}\left(\partial K \cap\left\{\epsilon 2^{-i-1} \leq R<2^{-i} \epsilon\right\}\right) \\
& \leq \sum_{i=0}^{\infty}\left(\epsilon 2^{-i-1}\right)^{-\alpha}(n-1) 2^{-i} \epsilon \mathscr{H}^{n-1}(\partial K) \\
& =\epsilon^{1-\alpha}(n-1) \frac{2^{\alpha}}{1-2^{\alpha-1}} \mathscr{H}^{n-1}(\partial K) .
\end{aligned}
$$

It follows that

$$
\begin{aligned}
\int_{B} R^{-\alpha} d \mathscr{H}^{n-1} & =\int_{B \cap\{R<\epsilon\}} R^{-\alpha} d \mathscr{H}^{n-1}+\int_{B \cap\{R \geq \epsilon\}} R^{-\alpha} d \mathscr{H}^{n-1} \\
& \leq \epsilon^{1-\alpha}(n-1) \frac{2^{\alpha}}{1-2^{\alpha-1}} \mathscr{H}^{n-1}(\partial K)+\epsilon^{-\alpha} \mathscr{H}^{n-1}(B) .
\end{aligned}
$$

We get the inequality of the lemma by choosing

$$
\epsilon:=\frac{1-2^{\alpha-1}}{2^{\alpha}(n-1)} \frac{\mathscr{H}^{n-1}(B)}{\mathscr{H}^{n-1}(\partial K)} .
$$


2.2. Hilbert geometries. The Hilbert distance between two distinct points $x$ and $y$ in int $K$ is defined by

$$
d(x, y):=\frac{1}{2}|\log [a, b, x, y]|,
$$

where $a$ and $b$ are the intersections of the line passing through $x$ and $y$ with the boundary $\partial K$, and $[a, b, x, y]$ denotes the cross-ratio (adopting the convention of [Bridson and Haefliger 1999]).

This distance is invariant under projective transformations. If $K$ is an ellipsoid, the Hilbert geometry on int $K$ is isometric to hyperbolic $n$-space.

Unbounded closed convex sets with nonempty interiors and not containing a straight line are projectively equivalent to convex bodies. Therefore, the definition of the distance naturally extends to the interiors of such convex sets. In particular, the convex sets bounded by parabolas are also isometric to the hyperbolic space.

Let us assume the origin $o$ lies inside the interior of $K$. We will write $B(r)$ for the metric ball of radius $r$ and centered at $o$. Its boundary, the metric sphere, will be denoted by $S(r)$. Let $a: \partial K \rightarrow \mathbb{R}_{+}$be defined by the equation $-a(p) p \in \partial K$, so the letter $a$ refers to the antipodal point. It is an easy exercise to check that metric spheres are parameterized by the boundary $\partial K$ as

$$
S(r)=\{\phi(p, r): p \in \partial K\}
$$

where

$$
\phi: \partial K \times \mathbb{R}_{+} \rightarrow \operatorname{int} K, \quad(p, r) \mapsto a \frac{e^{2 r}-1}{a e^{2 r}+1} p .
$$

The Hilbert distance comes from a Finsler metric on the interior of $K$. Given $x \in$ int $K$ and $v \in T_{x} V$, the Finsler norm of $v$ is given by

$$
\|v\|_{x}=\frac{1}{2}\left(\frac{1}{t_{1}}+\frac{1}{t_{2}}\right)
$$

where $t_{1}, t_{2}>0$ are such that $x \pm t_{i} v \in \partial K$. Again, we do not exclude that one of the $t_{i}$ is infinite. Equivalently, if $F_{x}$ is the Minkowski functional of $K-x$, then

$$
\|v\|_{x}=\frac{1}{2}\left(F_{x}(v)+F_{x}(-v)\right) .
$$

The Finsler metric makes it possible to measure the length of a differentiable curve $c: I \rightarrow$ int $K$ by

$$
l(c):=\int_{I}\left\|c^{\prime}(t)\right\|_{c(t)} d t
$$

It is less trivial to measure the area (or volume) of higher dimensional subsets of int $K$. In fact, different notions of volume are being used. The most important ones are the Busemann definition (which is equal to the Hausdorff $n$-dimensional measure) and the Holmes-Thompson definition. In the following, only properties 
of volumes in Finsler spaces (as defined in [Álvarez Paiva and Thompson 2004]) will be used:

- Vol is a Borel measure on int $K$ that is absolutely continuous with respect to Lebesgue measure.

- If $A \subset K \subset L$, where $K, L$ are compact convex sets, then the measure of $A$ with respect to $K$ is larger than the measure of $A$ with respect to $L$.

- If $K$ is an ellipsoid, then $\operatorname{Vol}(A)$ is the hyperbolic volume of $A$.

We will mainly investigate the following projective invariants of convex bodies.

Definition 2.5. The upper and lower volume entropies of $K$ are

$$
\overline{\operatorname{Ent}}(K):=\limsup _{r \rightarrow \infty} \frac{\log (\operatorname{Vol} B(r))}{r} \text { and } \quad \underline{\operatorname{Ent}}(K):=\liminf _{r \rightarrow \infty} \frac{\log (\operatorname{Vol} B(r))}{r} .
$$

If the upper and lower volume entropies of $K$ coincide, their common value is called the volume entropy of $K$ and is denoted by Ent $K$.

Note that these invariants are independent of the choice of the center and of the choice of the volume definition.

2.3. Busemann's density. For simplicity, we restrict ourselves to Busemann's volume, although all results remain true for every other choice of volume. The reason is that the proofs of the crucial Propositions 2.7 and 2.8 below do not use any particular property of Busemann's volume, but only the axioms satisfied by every definition of volume.

The density of Busemann's volume (with respect to some Lebesgue measure $\mathscr{L}$ ) is given by $\sigma(x)=\omega_{n} / \mathscr{L}\left(B_{x}\right)$, where $B_{x}$ is the tangent unit ball of the Finsler metric at $x$ and $\omega_{n}$ is the (Euclidean) volume of the unit ball in $\mathbb{R}^{n}$. The volume of a Borel subset $A \subset$ int $K$ is thus given by $\operatorname{Vol}(A)=\int_{A} \sigma d \mathscr{L}$.

We now state and prove some propositions concerning upper bounds and asymptotic behaviors of Busemann's densities for points that are close to the boundaries of particular convex sets. We will make use of an auxiliary inner product, calling $\mathscr{L}$ and $\mu$ the corresponding Lebesgue measure and volume $n$-form. Busemann densities are defined with this particular choice of measure.

Proposition 2.6. Let $K$ and $K^{\prime}$ be closed convex sets not containing any straight line and let $\sigma:$ int $K \rightarrow \mathbb{R}$ and $\sigma^{\prime}:$ int $K^{\prime} \rightarrow \mathbb{R}$ be their corresponding Busemann densities. Let $p \in \partial K$, let $E_{0}$ be a support hyperplane of $K$ at $p$, and let $E_{1}$ be a hyperplane parallel to $E_{0}$ intersecting $K$. Suppose that $K$ and $K^{\prime}$ have the same intersection with the strip between $E_{0}$ and $E_{1}$ (in particular $p \in \partial K^{\prime}$ ). Then

$$
\lim _{y \rightarrow p} \sigma(y) / \sigma^{\prime}(y)=1 .
$$


Proof. Let $d$ be the distance between $E_{0}$ and $E_{1}$, and let $\left(y_{i}\right)$ be a sequence of points of int $K$ converging to $p$. We may suppose that the distance $d_{i}$ between $y_{i}$ and $E_{0}$ is strictly less than $d$. For every fixed point $y_{i}$ and nonzero tangent vector $v \in T_{y_{i}} K$, let $t_{1}, t_{2} \in \mathbb{R}_{+} \cup\{\infty\}$ be such that $y_{i} \pm t_{1,2} v \in \partial K$; let $t_{1}^{\prime}$ and $t_{2}^{\prime}$ be the corresponding numbers for $K^{\prime}$. Since at least one of $y_{i}+t_{1} v$ and $y_{i}-t_{2} v$ is inside the strip, say $y_{i}+t_{1} v$, we must have $t_{1}=t_{1}^{\prime}$.

Either $t_{2}=t_{2}^{\prime}$ and $\|v\|_{i}=\|v\|_{i}^{\prime}$, or $t_{2} \neq t_{2}^{\prime}$, in which case

$$
\frac{t_{1}}{t_{2}}, \frac{t_{1}^{\prime}}{t_{2}^{\prime}} \leq \frac{d_{i}}{d-d_{i}} \text {. }
$$

Therefore,

$$
\frac{d-d_{i}}{d} \leq \frac{\|v\|_{i}}{\|v\|_{i}^{\prime}}=\frac{1+\left(t_{1} / t_{2}\right)}{1+\left(t_{1}^{\prime} / t_{2}^{\prime}\right)} \leq \frac{d}{d-d_{i}},
$$

which shows that, as functions on $\mathbb{R} P^{n-1}$, the $\|\cdot\|_{i} /\|\cdot\|_{i}^{\prime}$ uniformly converge to 1 . Hence, for every $\epsilon$ and every $i$ large enough, $(1-\epsilon) B_{y_{i}} \subset B_{y_{i}}^{\prime} \subset(1+\epsilon) B_{y_{i}}$, which implies the convergence of $\sigma / \sigma^{\prime}$ to 1 .

Proposition 2.7. Let $V=\mathbb{R}^{n}$ with its usual scalar product. Let $P$ be the convex set bounded by the parabola $y=\sum_{i=1}^{n-1}\left(c_{i} / 2\right) x_{i}^{2}$, with $c_{1}, \ldots, c_{n-1}>0$. Then

$$
\sigma(0, \ldots, 0,1-\lambda)=\frac{\sqrt{c}}{(2(1-\lambda))^{(n+1) / 2}}, \quad \text { where } c=\prod_{i=1}^{n-1} c_{i} .
$$

Proof. By the invariance of the Hilbert metric under projective transformations, the tangent unit sphere at any point of int $P$ is an ellipse. At the point $(0, \ldots, 0$, $1-\lambda)$, the symmetry implies that the principal axes of this ellipse are parallel to the coordinate axes. Hence $\sigma=1 / \prod_{i=1}^{n} l_{i}$, where the $l_{i}$ for $i=1, \ldots, n$ are the Euclidean lengths of the principal half-axes.

Now $l_{i}=\sqrt{2(1-\lambda) / c_{i}}$ for $i=1, \ldots, n-1$ and $l_{n}=2(1-\lambda)$.

Proposition 2.8. Assume the origin o is inside int $K$. For a smooth point $p$ of $\partial K$, let $n(p)$ be the outward normal vector and let $k(p)$ be the Gauss curvature of $\partial K$ at $p$. Then

$$
\lim _{\lambda \rightarrow 1} \sigma(\lambda p)(1-\lambda)^{(n+1) / 2}=\frac{\sqrt{k(p)}}{(2\langle p, n(p)\rangle)^{(n+1) / 2}} .
$$

Proof. Let us choose a frame $\left(p ; v_{1}, \ldots, v_{n-1}, v_{n}\right)$, where $v_{1}, \ldots, v_{n-1} \in T_{p} \partial K$ are unit vectors tangent to the principal curvature directions of $\partial K$ at $p$ and $v_{n}=-p$. In these coordinates, the boundary of $K$ is locally the graph of a function

$$
y=\sum_{i=1}^{n-1}\left(c_{i} / 2\right) x_{i}^{2}+R(|x|),
$$


with $R(|x|)=o\left(|x|^{2}\right)$ and $c_{1}, \ldots, c_{n-1} \geq 0$. We set $c:=\prod_{i=1}^{n-1} c_{i}$. Then a short computation shows that $d x_{1} \wedge \cdots \wedge d x_{n-1} \wedge d y=\mu / m$, where $\mu$ is the Euclidean $n$-form and $m:=\mu\left(v_{1}, \ldots, v_{n}\right)=\langle p, n(p)\rangle$. Also, the Gauss curvature at $p$ is given by $k(p)=c m^{n-1}$.

Let us fix $\epsilon>0$. Locally, the parabola defined by $y=\sum_{i=1}^{n-1} \frac{1}{2}\left(c_{i}+\epsilon\right) x_{i}^{2}$ lies inside $K$. Cutting it with some horizontal hyperplane, we obtain a convex body $K^{\prime}$ inside $K$. In particular, the metric of $K^{\prime}$ is greater than or equal to the metric of $K$; hence, $\sigma^{\prime}(\lambda p) \geq \sigma(\lambda p)$ for $\lambda$ near 1 .

Then by Propositions 2.6 and 2.7,

(15) $\limsup _{\lambda \rightarrow 1} \sigma(\lambda p)(1-\lambda)^{(n+1) / 2} \leq \lim _{\lambda \rightarrow 1} \sigma^{\prime}(\lambda p)(1-\lambda)^{(n+1) / 2}=\frac{\sqrt{\prod_{i=1}^{n-1}\left(c_{i}+\epsilon\right)}}{2^{(n+1) / 2} m}$.

Since $\sigma>0$, this already settles the case $k=c=0$, since $\epsilon$ was arbitrarily small.

If $c>0$ and $0<\epsilon<\min \left\{c_{1}, \ldots, c_{n-1}\right\}$, the parabola $P$ defined by

$$
y=\sum_{i=1}^{n-1} \frac{c_{i}-\epsilon}{2} x_{i}^{2}
$$

locally contains $K$. Cutting it with some horizontal hyperplane, we obtain a convex body $K^{\prime}$ inside $P$. Again by Propositions 2.6 and 2.7,

(16) $\liminf _{\lambda \rightarrow 1} \sigma(\lambda p)(1-\lambda)^{(n+1) / 2} \geq \liminf _{\lambda \rightarrow 1} \sigma^{\prime}(\lambda p)(1-\lambda)^{(n+1) / 2}=\frac{\sqrt{\prod_{i=1}^{n-1}\left(c_{i}-\epsilon\right)}}{2^{(n+1) / 2} m}$.

From (15) and (16) (with $\epsilon \rightarrow 0$ ) we get

$$
\lim _{\lambda \rightarrow 1} \sigma(\lambda p)(1-\lambda)^{(n+1) / 2}=\frac{\sqrt{c}}{2^{(n+1) / 2} m} .
$$

Section 3 will start with the proof of a slight and somewhat technical refinement of our second main theorem. To state it precisely, we need to introduce the pseudoGauss curvature of the boundary of a convex set $K$ in $\mathbb{R}^{n}$.

For a smooth point $p \in \partial K$, let $n(p)$ be the outward normal of $\partial K$ at $p$. For each unit vector $e \in T_{p} \partial K$, let $H_{e}(p)$ be the affine plane containing $p$ and directed by the vectors $e$ and $n(p)$. We define $R_{e}$ as the radius of the biggest disc containing $p$ inside $K_{e}:=K \cap H_{e}(p)$.

Definition 2.9. The pseudo-Gauss curvature $\bar{k}(p)$ of $\partial K$ at $p$ is the minimum of the numbers $\prod_{i=1}^{n-1} R_{e_{i}}(p)^{-1}$, where $e_{1}, \ldots, e_{n-1}$ ranges over all orthonormal bases of $T_{p} \partial K$. 
Proposition 2.10. Let $V$ be a Euclidean vector space of dimension $n$. Let $K$ be a convex body containing the unit ball $B$. Then for $\frac{1}{2} \leq \lambda<1$ and $p \in \partial K$,

$$
\sigma(\lambda p) \leq \frac{\omega_{n} n !}{2^{n}(1-\lambda)^{(n+1) / 2}} \bar{k}(p)^{1 / 2} .
$$

Proof. We use the same notation as in the definition of $\bar{k}$. We may suppose that $R_{i}:=R_{e_{i}}(p)>0$ for all $i$; otherwise the statement is trivial. By the definition of $R_{i}$, there is a 2-disc $B_{i}(p)$ of radius $R_{i}$ inside $K_{e_{i}}$ containing $p$. Let us denote by $B\left(e_{i}\right)$ the intersection of $B$ with the affine plane $p+H_{e_{i}}$. Since $B\left(e_{i}\right)$ and $B_{i}(p) \subset K$,

$$
\hat{C}_{i}:=\operatorname{conv}\left(B\left(e_{i}\right) \times\{0\} \cup B_{i}(p) \times\{1\}\right) \subset K_{e_{i}} \times[0,1] .
$$

Note that $\hat{C}_{i}$ is a truncated cone. Let $E_{i}$ be the plane containing the line that is parallel to $T_{p} \partial K_{e_{i}}$ and that passes through the points $o \times\{0\}$ and $p \times\{1\}$. With $\pi: V \times[0,1] \rightarrow V$ the projection on the first component, $C_{i}:=\pi\left(E_{i} \cap \hat{C}_{i}\right) \subset K$ is bounded by a truncated conic.

In the nonorthogonal frame $\left(o ; p, e_{i}\right), C_{i}$ is given by

$$
\left(2 R_{i}-1\right) x^{2}+2\left(1-R_{i}\right) x+y_{1}^{2} \leq 1 \quad \text { for } 0 \leq x \leq 1 .
$$

Now let $C$ be the convex hull of the union of the $C_{i}$. Then the polytope $P$ with vertices

$$
\left(\lambda, 0, \ldots, \pm \sqrt{(1-\lambda)\left(2 \lambda R_{i}-\lambda+1\right)}, 0, \ldots, 0\right), \quad(1, \overrightarrow{0}), \quad(2 \lambda-1, \overrightarrow{0})
$$

lies inside $C$, with all but the last vertex being on the boundaries of the $C_{i}$.

Its volume is given by

$$
\begin{aligned}
\mathscr{L}(P) & =\frac{2^{n}\langle p, n(p)\rangle}{n !}(1-\lambda)^{(n+1) / 2} \prod_{i=1}^{n-1}\left(2 \lambda R_{i}-\lambda+1\right)^{1 / 2} \\
& \geq \frac{2^{n}}{n !}(1-\lambda)^{(n+1) / 2}\left(R_{1} \cdot R_{2} \cdots R_{n-1}\right)^{1 / 2}=\frac{2^{n}}{n !}(1-\lambda)^{(n+1) / 2} \bar{k}^{-1 / 2}(p) .
\end{aligned}
$$

The factor $\langle p, n(p)\rangle$ in the first line appears because our coordinate system is not orthonormal. Since the unit ball is contained in $K$, this factor is at least 1 .

From $P \subset C \subset K$ and the fact that $P$ is centered at $\lambda p$, we deduce that

$$
\sigma(\lambda p) \leq \frac{\omega_{n}}{\mathscr{L}(P)} \leq \frac{\omega_{n} n !}{2^{n}}(1-\lambda)^{-(n+1) / 2} \bar{k}^{1 / 2}(p) .
$$

The next proposition will be needed in the construction of a convex body with entropy between 0 and 1 .

Proposition 2.11. Let $K=o a b$ be a triangle with $1 \leq o a$ and $o b \leq 2$, such that the distance from $o$ to the line passing through $a$ and $b$ is at least 1 . Let $p$ be $a$ 
point in the interior of the side $a b$ and suppose that $\min \{a p, b p\} \geq \epsilon>0$. Then for $\lambda \geq 1 / 2$, Busemann's density of $K$ at $\lambda p$ is bounded above by

$$
\sigma(\lambda p) \leq 32 \pi \max \left\{\frac{1}{\epsilon(1-\lambda)}, \frac{1}{\epsilon^{2}}\right\} .
$$

Proof. The hypothesis on the triangle implies that $\sin (a b o), \sin (b a o) \geq 1 / 2$.

Let $a^{\prime}$ be the intersection with $o b$ of the line passing through $a$ and $z:=\lambda p$, and define $b^{\prime}$ similarly.

The unit tangent ball at $z$ is a hexagon centered at $z$. The length of one of its half-diagonals is the harmonic mean of $z a$ and $z a^{\prime}$; the length of the second halfdiagonal is the harmonic mean of $z b$ and $z b^{\prime}$; and the third half-diagonal has length

$$
\frac{2 o p}{\frac{1}{\lambda}+\frac{1}{1-\lambda}} \geq 1-\lambda \text {. }
$$

An easy geometric argument shows that

$$
z a^{\prime}, z b \geq \frac{1}{2} p b \sin (a b o) \geq \frac{1}{4} \epsilon \quad \text { and } \quad z a, z b^{\prime} \geq \frac{1}{2} p a \sin (b a o) \geq \frac{1}{4} \epsilon .
$$

The area $A$ of the hexagon is at least half of the minimal product of two of its half-diagonals; hence, $A \geq \min \left\{\frac{1}{8} \epsilon(1-\lambda), \frac{1}{32} \epsilon^{2}\right\}$.

2.4. Volume entropy of spheres. By definition, the entropy controls the volume growth of metric balls in Hilbert geometries. We show in this section that it coincides with the growth of areas of metric spheres. Again, there are several definitions of area of hypersurfaces in Finsler geometry. For simplicity, we consider Busemann's definition, which gives the Hausdorff $(n-1)$-measure of these hypersurfaces.

Lemma 2.12 (rough monotonicity of area). There exist a monotone function $f$ and a constant $C_{1}>1$ such that for all $r>0$,

$$
C_{1}^{-1} f(r) \leq \operatorname{Area}(S(r)) \leq C_{1} f(r) .
$$

Proof. Let $f(r)$ be the Holmes-Thompson area of $S(r)$. Since all area definitions agree up to some universal constant, inequality (19) is trivial. It remains to show that $f$ is monotone.

If $\partial K$ is $C^{2}$ with everywhere positive Gaussian curvature, then the tangent unit spheres of the Finsler metric are quadratically convex. According to [Álvarez Paiva and Fernandes 1998, Theorem 1.1 and Remark 2], there exists a Crofton formula for the Holmes-Thompson area, from which the monotonicity of $f$ easily follows.

Such smooth convex bodies are dense in the set of all convex bodies for the Hausdorff topology; see for example [Hörmander 2007, Lemma 2.3.2]. By approximation, it follows that $f$ is monotone for arbitrary $K$. 
Lemma 2.13 (coarea inequalities). There exists a constant $C_{2}>1$ such that

$$
C_{2}^{-1} \operatorname{Area}(S(r)) \leq \frac{\partial}{\partial r} \operatorname{Vol}(B(r)) \leq C_{2} \operatorname{Area}(S(r)) \quad \text { for all } r>0 .
$$

Proof. Let $\mu:=\sigma d x_{1} \wedge \cdots \wedge d x_{n}$ be the volume form, and let $\alpha$ be the (n-1)-form on $S(r)$ whose integral equals the area.

Since

$$
\operatorname{Vol}(B(r))=\int_{0}^{r} \int_{S(s)} i_{\partial_{r}} \mu d s,
$$

where $\partial_{r}$ at $\lambda p \in S(s)$ is the tangent vector multiple of $\overrightarrow{o p}$ with unit Finsler norm, we have to compare $i_{\partial_{r}} \mu$ and $\alpha$.

We will assume that $S(r)$ is differentiable at $\lambda p$. The section of the unit tangent ball by the tangent space $T_{\lambda p} S(r)$ will be called $\gamma$. By the definition of Busemann area, the area of $\gamma$ measured with the form $\alpha$ is the constant $\alpha(\gamma)=\omega_{n-1}$.

In the same way, calling $\Gamma$ the half unit ball containing $\partial_{r}$ and bounded by $\gamma$, one has $\mu(\Gamma)=\frac{1}{2} \omega_{n}$.

Since $\Gamma$ is convex, it contains the cone with base $\gamma$ and vertex $\partial_{r}$. Therefore,

$$
\frac{1}{n} i_{\partial_{r}} \mu(\gamma) \leq \frac{1}{2} \omega_{n} .
$$

By Brunn's theorem (see for example [Koldobsky 2005, Theorem 2.3]), the sections of the tangent unit ball with hyperplanes parallel to $\gamma$ have an area less than or equal to the area of $\gamma$. Also the tangent unit ball has a supporting hyperplane at $\partial_{r}$ which is parallel to $\gamma$. Therefore, by Fubini's theorem, the cylinder $\gamma \times\left([0,1] \cdot \partial_{r}\right)$ has a volume greater than or equal to the volume of $\Gamma$ (even if it generally does not contain $\Gamma$ ). Hence,

$$
\frac{1}{2} \omega_{n} \leq i_{\partial_{r}} \mu(\gamma) .
$$

Inequalities (20) and (21) give

$$
\frac{1}{2} \frac{\omega_{n}}{\omega_{n-1}} \alpha(\gamma) \leq i_{\partial_{r}} \mu(\gamma) \leq \frac{n}{2} \frac{\omega_{n}}{\omega_{n-1}} \alpha(\gamma),
$$

from which the result easily follows.

Theorem 2.14. The spherical entropy coincides with the entropy. More precisely, $\limsup _{r \rightarrow \infty} \frac{\log \operatorname{Area}(S(r))}{r}=\overline{\operatorname{Ent}} K \quad$ and $\quad \liminf _{r \rightarrow \infty} \frac{\log \operatorname{Area}(S(r))}{r}=\underline{\text { Ent }} K$.

Proof. For convenience, let $V(r):=\operatorname{Vol} B(r)$ and $A(r):=$ Area $S(r)$.

Using the previous two lemmas, one has, for all $r>0$,

$$
\begin{aligned}
V(r)=\int_{0}^{r} V^{\prime}(s) d s & \leq C_{2} \int_{0}^{r} A(s) d s \leq C_{1} C_{2} \int_{0}^{r} f(s) d s \\
& \leq C_{1} C_{2} f(r) r \leq C_{1}^{2} C_{2} A(r) r .
\end{aligned}
$$


It follows that

$$
\overline{\text { Ent }} K=\limsup _{r \rightarrow \infty} \frac{\log V(r)}{r} \leq \limsup _{r \rightarrow \infty} \frac{\log C_{1}^{2} C_{2} A(r) r}{r}=\limsup _{r \rightarrow \infty} \frac{\log \operatorname{Area}(S(r))}{r} \text {. }
$$

Similarly, for each $\epsilon>0$,

$$
\begin{aligned}
V(r(1+\epsilon)) & =\int_{0}^{r(1+\epsilon)} V^{\prime}(s) d s \geq C_{1}^{-1} C_{2}^{-1} \int_{0}^{r(1+\epsilon)} f(s) d s \\
& \geq C_{1}^{-1} C_{2}^{-1} \int_{r}^{r(1+\epsilon)} f(s) d s \geq C_{1}^{-1} C_{2}^{-1} f(r) r \epsilon \geq C_{1}^{-2} C_{2}^{-1} A(r) r \epsilon,
\end{aligned}
$$

and hence

$$
\begin{aligned}
(1+\epsilon) \overline{\operatorname{Ent}} K & =(1+\epsilon) \limsup _{r \rightarrow \infty} \frac{\log V(r(1+\epsilon))}{r(1+\epsilon)} \\
& \geq \limsup _{r \rightarrow \infty} \frac{\log C_{2}^{-1} C_{1}^{-2} A(r) r \epsilon}{r}=\limsup _{r \rightarrow \infty} \frac{\log \operatorname{Area}(S(r))}{r} .
\end{aligned}
$$

Letting $\epsilon \rightarrow 0$ gives the first equality. The second one follows in a similar way.

\section{Entropy bounds}

3.1. Upper entropy bound in arbitrary dimension. Our second main theorem will follow from the next result.

Theorem 3.1. Let $K$ be an $n$-dimensional convex body and $o \in$ int $K$. For a point $p \in \partial K$, we denote by $\bar{k}(p)$ its pseudo-Gauss curvature as in Definition 2.9. If

$$
\int_{\partial K} \bar{k}^{1 / 2}(p) d p<\infty
$$

then

$$
\lim _{r \rightarrow \infty} \frac{\operatorname{Vol} B(o, r)}{\sinh ^{n-1} r}=\frac{1}{n-1} \mathscr{A}_{p}(K) .
$$

In particular, $\overline{\text { Ent }} K \leq n-1$, and if $\mathscr{A}_{p}(K) \neq 0$, then $\overline{\operatorname{Ent}} K=n-1$.

Proof. Using the parameterization (11), the volume of metric balls is given by

$$
\operatorname{Vol}(B(r))=\int_{0}^{r} \int_{\partial K} F(p, r) d \mathscr{H}^{n-1},
$$

where $F(p, r):=\sigma(\phi(p, r)) \operatorname{Jac} \phi(p, r)$.

The Jacobian may be explicitly computed:

$$
\operatorname{Jac} \phi(p, r)=\frac{\left(e^{2 r}-1\right)^{n-1} e^{2 r}}{\left(a e^{2 r}+1\right)^{n+1}} 2 a^{n}(1+a)\langle p, n(p)\rangle .
$$


In particular,

$$
\lim _{r \rightarrow \infty} e^{2 r} \operatorname{Jac} \phi(p, r)=2(1+a)\langle p, n(p)\rangle / a .
$$

On the other hand, for each smooth boundary point $p$ we have, by Proposition 2.8,

$$
\lim _{r \rightarrow \infty} \frac{\sigma(\phi(p, r))}{e^{(n+1) r}}=\frac{\sqrt{k(p)}}{(2\langle p, n(p)\rangle)^{(n+1) / 2}} \frac{a^{(n+1) / 2}}{(1+a)^{(n+1) / 2}} .
$$

Then, by Proposition 2.10 and the hypothesis (22),

$$
\begin{aligned}
\lim _{r \rightarrow \infty} \frac{1}{e^{(n-1) r}} & \int_{\partial K} F(p, r) d \mathscr{H}^{n-1}=\int_{\partial K} \lim _{r \rightarrow \infty} \frac{F(p, r)}{e^{(n-1) r}} d \mathscr{H}^{n-1} \\
& =\int_{\partial K} \lim _{r \rightarrow \infty} \frac{\sigma(\phi(p, r))}{e^{(n+1) r}} \lim _{r \rightarrow \infty} e^{2 r} \operatorname{Jac} \phi(p, r) d \mathscr{H}^{n-1} \\
& =\int_{\partial K} \frac{\sqrt{k(p)}}{(2\langle p, n(p)\rangle)^{(n-1) / 2}}\left(\frac{a}{1+a}\right)^{n-1 / 2} d \mathscr{H}^{n-1}=\frac{1}{2^{n-1}} \mathscr{A}_{p}(K) .
\end{aligned}
$$

By L'Hôpital's rule, we get

$$
\lim _{r \rightarrow \infty} \frac{\operatorname{Vol}(B(r))}{e^{(n-1) r}}=\lim _{r \rightarrow \infty} \frac{\int_{0}^{r} \int_{\partial K} F(p, s) d \mathscr{H}^{n-1} d s}{(n-1) \int_{0}^{r} e^{(n-1) s} d s}=\frac{1}{2^{n-1}(n-1)} \mathscr{A}_{p}(K) .
$$

Remark. The metric balls $B(r)$ are projective invariants of $K$. There is an affine version of the previous theorem using the affine balls $B_{a}(r):=\tanh (r) K$ (where multiplication is with respect to the center $o$ ). Under the same assumptions as in Theorem 3.1, we obtain that

$$
\lim _{r \rightarrow \infty} \frac{\operatorname{Vol} B_{a}(r)}{e^{(n-1) r}}=\frac{1}{2^{n-1}(n-1)} \mathscr{A}_{a}(K),
$$

where $\mathscr{A}_{a}(K)$ is the centroaffine area (see Section 4). The proof goes as the one above by replacing the function $a$ by 1 .

Corollary 3.2. Suppose $K$ is an $n$-dimensional convex body of class $C^{1,1}$. Then

$$
\text { Ent } K=n-1 \text {. }
$$

Proof. For any $p \in \partial K, R(p)$ is the biggest radius of a ball in $K$ containing $p$. By Proposition 2.2, there exists a constant $R>0$ such that $R(p) \geq R$ for all $p \in \partial K$. It follows that the hypothesis (22) is satisfied and therefore Ent $K \leq n-1$.

The Gauss map $\mathscr{G}: \partial K \rightarrow S^{n-1}$ is well defined and continuous. As a consequence of [Hug 1999a, Theorem 2.3] and [Hug 1998, Equation 2.7], the standard measure on the unit sphere is the push-forward of $k \cdot d \mathscr{H}^{n-1}$, that is,

$$
\mathscr{G}_{*}\left(\left.k \cdot d \mathscr{H}^{n-1}\right|_{\partial K}\right)=\left.d \mathscr{H}^{n-1}\right|_{S^{n-1}},
$$


and hence the curvature has a positive integral. Therefore, $\mathscr{A}_{p}(K)>0$, and (23) implies that Ent $K=n-1$.

Corollary 3.3. If $K$ is an arbitrary $n$-dimensional convex body with $\mathscr{A}_{p}(K) \neq 0$, then Ent $K \geq n-1$.

Proof. Arguing as in the proof of Theorem 3.1 and using Fatou's lemma instead of the dominated convergence theorem gives the result.

3.2. The plane case. Let us now assume that $n=2$. By Theorem 2.3 , the hypothesis (22) is satisfied for each convex body $K$. Therefore

$$
\overline{\text { Ent }} K \leq 1
$$

and

$$
\lim _{r \rightarrow \infty} \frac{\operatorname{Vol} B(o, r)}{\sinh r}=\mathscr{A}_{p}(K) .
$$

Next, we are going to prove a better bound for $\overline{\operatorname{Ent}} K$. To state our main result, we need to recall some basic notions of measure theory in a Euclidean space and refer to [Mattila 1995] for details. For a nonempty bounded set $A$, let $N(A, \epsilon)$ be the minimal number of $\epsilon$-balls needed to cover $A$. Then the upper Minkowski dimension of $A$ is defined as

$$
\overline{\operatorname{dim}} A:=\inf \left\{s: \lim \sup _{\epsilon \rightarrow 0} N(A, \epsilon) \epsilon^{s}=0\right\} .
$$

One should note that this dimension is invariant under bi-Lipschitz maps. In particular, it does not depend on a particular choice of inner product, and it is invariant under projective maps provided the considered subsets are bounded.

Recall that a point $p \in K$ is called extremal if it is not a convex combination of other points of $K$. The set of extremal points is a subset of $\partial K$, which we denote by ex $K$.

First main theorem. Let $K$ be a plane convex body, and let $d$ be the upper Minkowski dimension of $\mathrm{ex}$. Then the entropy of $K$ is bounded by

$$
\overline{\text { Ent }} K \leq \frac{2}{3-d} \leq 1 \text {. }
$$

Proof. Since the entropy is independent of the choice of the center, we may suppose that the Euclidean unit ball around $o$ is the maximum volume ellipsoid inside $K$. Then $K$ is contained in the ball of radius 2 [Barvinok 2002].

Set $\epsilon:=e^{-\alpha r}$, where $\alpha \leq 1$ will be fixed later. Divide the boundary of $K$ into two parts:

$$
\partial K=\mathscr{B} \cup \mathscr{G},
$$

where $\mathscr{B}$ (the bad part) is the closed $\epsilon$-neighborhood around the set of extremal points of $K$ and $\mathscr{G}$ (the good part) is its complement. 
Using Proposition 2.4 and equalities (24) and (25), we get an upper bound for large values of $r$ :

$$
\int_{r / 2}^{r} \int_{\mathscr{乃}} \sigma(\phi(p, s)) \operatorname{Jac} \phi(p, s) d \mathscr{H}^{1} d s \leq O\left(e^{r} \sqrt{\mathscr{H}^{1}(\mathscr{B})}\right) .
$$

Next, let $p \in \mathscr{G}$. The endpoints of the maximal segment in $\partial K$ containing $p$ are extremal points of $K$ and hence of distance at least $\epsilon$ from $p$. Therefore $K$ contains a triangle as in Proposition 2.11, and if $s \geq r / 2$, and $r$ is sufficiently large,

$$
\sigma(\phi(p, s))=\sigma(\lambda \cdot p) \leq 32 \max \left\{\frac{1}{\epsilon(1-\lambda)}, \frac{1}{\epsilon^{2}}\right\}=\frac{32}{\epsilon(1-\lambda)} .
$$

Integrating this from $r / 2$ to $r$ yields

$$
\int_{r / 2}^{r} \int_{\mathscr{G}} \sigma(\phi(p, s)) \operatorname{Jac} \phi(p, s) d \mathscr{H}^{1} d s=O\left(e^{\alpha r}\right) .
$$

Let $d$ be the upper Minkowski dimension of the set of extremal points of $K$. Then, for each $\eta>0, N($ ex $K, \epsilon)=o\left(\epsilon^{-d-\eta}\right)$ as $\epsilon \rightarrow 0$. By the definition of $N$, there is a covering of ex $K$ by $N(\operatorname{ex} K, \epsilon)$ balls of radius $\epsilon$. Hence there is a covering of $\mathscr{B}$ by $N(\mathrm{ex} K, \epsilon)$ balls with radius $2 \epsilon$. The intersection of a $2 \epsilon$-ball with $\partial K$ has length less than $4 \pi \epsilon$. It follows that $\mathscr{H}^{1}(\mathscr{B})=o\left(\epsilon^{-d-\eta+1}\right)$. Since the volume of $B(r / 2)$ is bounded by $O\left(e^{r / 2}\right)$ (see (27)), the volume of $B(r)$ is bounded by

$$
\begin{aligned}
\operatorname{Vol} B(r)= & \operatorname{Vol} B(r / 2)+\int_{r / 2}^{r} \int_{\mathscr{B}} \sigma(\phi(p, s)) \operatorname{Jac} \phi(p, s) d \mathscr{H}^{1} d s \\
& +\int_{r / 2}^{r} \int_{\mathscr{G}} \sigma(\phi(p, s)) \operatorname{Jac} \phi(p, s) d \mathscr{H}^{1} d s \\
= & O\left(e^{r / 2}\right)+O\left(e^{r(1-(\alpha(1-d-\eta)) / 2)}\right)+O\left(e^{\alpha r}\right) .
\end{aligned}
$$

We fix $\alpha$ such that $1-\alpha(1-d-\eta) / 2=\alpha$, that is, $\alpha:=2 /(3-d-\eta)>2 / 3$. Then $\operatorname{Vol} B(r)=O\left(e^{\alpha r}\right)$, which implies that the (upper) entropy of $K$ is bounded by $\alpha$. Since $\eta>0$ was arbitrary, the result follows.

3.3. An example of noninteger entropy. We will construct a plane convex body with piecewise affine boundary whose entropy is strictly between 0 and 1 .

Let us choose a real number $s>2$ and set $\alpha_{i}:=C_{s} / i^{s}$, where $C_{s}>0$ is sufficiently small, such that $3 \sum_{i=1}^{\infty} \alpha_{i}<\pi$. We consider a centrally symmetric sequence $E$ of points on $S^{1}$ such that the angles between consecutive points are $\alpha_{1}, \alpha_{1}, \alpha_{1}, \alpha_{2}, \alpha_{2}, \alpha_{2}, \ldots$ (each angle appearing three times).

Theorem 3.4. The entropy of $K=\operatorname{conv}(E)$ is bounded by

$$
0<\frac{1}{s} \leq \underline{\text { Ent }} K \leq \overline{\operatorname{Ent}} K \leq \frac{2 s-2}{3 s-4}<1 .
$$


Proof for lower bound. The unit sphere of radius $r$ in the Hilbert geometry $K$ is $\tanh r K$ and consists of an infinite number of segments.

An easy geometric computation shows that the middle segment $S_{i}(r)$ corresponding to $\alpha:=\alpha_{i}$ has for each $r \geq 0$ length bounded from below by

$$
l\left(S_{i}(r)\right) \geq \log \left(\frac{2 \tanh r}{1-\tanh r} \tan (\alpha / 2) \sin (\alpha)+1\right) .
$$

Set $i_{0}(r):=\left\lfloor\left(2 C_{s}\right)^{1 / s} e^{r / s}\right\rfloor$. Then, for sufficiently large $r$,

$$
\frac{2 \tanh r}{1-\tanh r} \tan \left(\alpha_{i} / 2\right) \sin \left(\alpha_{i}\right) \leq 1 \quad \text { for all } i \geq i_{0}(r) \text {. }
$$

By the concavity of the $\log$-function, we have $\log (1+x) \geq x \log 2 \geq x / 2$ for $0 \leq x \leq 1$. Therefore

$$
l(S(r)) \geq \sum_{i=i_{0}}^{\infty} \frac{\tanh r}{1-\tanh r} \tan \left(\alpha_{i} / 2\right) \sin \left(\alpha_{i}\right) .
$$

For sufficiently large $r$, the first factor is bounded from below by $e^{2 r} / 4$, while the second is bounded from below by $\alpha_{i}^{2}$. We thus get

$$
l(S(r)) \geq \frac{e^{2 r}}{4} \sum_{i=i_{0}}^{\infty} \alpha_{i}^{2}=C_{s}^{2} \frac{e^{2 r}}{4} \sum_{i=i_{0}}^{\infty} \frac{1}{i^{2 s}} \geq C_{s}^{2} \frac{e^{2 r}}{4} \int_{i_{0}}^{\infty} \frac{1}{x^{2 s}} d x=C_{s}^{2} \frac{e^{2 r}}{4(2 s-1) i_{0}^{2 s-1}} .
$$

Replacing our explicit value for $i_{0}$ gives $l(S(r)) \geq C e^{r / s}$ for sufficiently large $r$ and some constant $C$ (again depending on $s$ ). Hence Ent $K \geq 1 / s$.

Proof for upper bound. For the upper bound in the statement, we apply our first main theorem. For this, we have to find an upper bound on the Minkowski dimension of ex $K=E$.

Since the Minkowski dimension is invariant under bi-Lipschitz maps, we may replace distances on the unit circle by angular distances.

$E$ has two accumulation points $\pm x_{0}$. For $\epsilon>0$, let $N(\epsilon)$ be the number of $\epsilon$-balls needed to cover $E$. We take one such ball around $\pm x_{0}$ and one further ball for each point in $E$ not covered by these two balls.

The three points corresponding to the angle $\alpha_{i}$ are certainly in the $\epsilon$-neighborhood of $\pm x_{0}$, provided that $3 \sum_{j=i}^{\infty} \alpha_{j} \leq \epsilon$.

Now we compute

$$
\sum_{j=i}^{\infty} \alpha_{j}=C_{s} \sum_{j=i}^{\infty} \frac{1}{j^{s}} \leq C_{s} \int_{i-1}^{\infty} \frac{1}{x^{s}} d x=\frac{C_{s}}{s-1} \frac{1}{(i-1)^{s-1}} .
$$

It follows that all $i$ satisfying $i \geq i_{0}:=\left(3 C_{s} /(s-1)\right)^{1 /(s-1)} \epsilon^{1 /(1-s)}+1$ also satisfy the inequality above, and hence $N(\operatorname{ex} K, \epsilon) \leq 6 i_{0}+2 \leq C \epsilon^{-1 /(s-1)}$. 
It follows that the upper Minkowski dimension is not larger than $1 /(s-1)$. The upper bound of First main theorem gives

$$
\overline{\text { Ent }} K \leq \frac{2 s-2}{3 s-4} .
$$

\section{Centroprojective and centroaffine areas}

In this section, we will take a closer look at the centroprojective area, which was introduced (in a nonintrinsic way) in the definition on page 204.

4.1. Basic definitions and properties. Geometrically speaking, both centroaffine and centroprojective areas are Riemannian volumes of the boundary $\partial K$.

We first give intrinsic definitions of the centroaffine metric and area. Let $K$ be a convex body with a distinguished interior point, which we may suppose to be the origin $o$ of $V$. The Minkowski functional of $K$ is the unique positive function $F$ that is homogeneous of degree 1 and whose level set at height 1 is the boundary $\partial K$. This function is convex and, according to Alexandroff's theorem, has almost everywhere a quadratic approximation.

Definition 4.1. Let $v$ be a tangent vector to $\partial K$ at a smooth point $p$. Then the centroaffine seminorm of $v$ is $\|v\|_{a}:=\sqrt{\operatorname{Hess}_{p} F(v, v)}$.

The square of the centroaffine seminorm is a quadratic function on the tangent, and hence we may define as usual a volume form, say $\omega_{a}$ (which vanishes if $\|\cdot\|_{a}$ is not definite).

Definition 4.2. The centroaffine area of $K$ is $\mathscr{A}_{a}(K):=\int_{\partial K}\left|\omega_{a}\right|$.

It easily follows from the definitions that the centroaffine area is indeed an affine invariant of pointed convex bodies. Moreover, it is finite and vanishes on polytopes. The next proposition relates our definitions with the classical ones; its proof is a straightforward computation.

Proposition 4.3. If the space is equipped with a Euclidean inner product, then the centroaffine area is given by

$$
\mathscr{A}_{a}(K)=\int_{\partial K} \frac{\sqrt{k}}{\langle n, p\rangle^{(n-1) / 2}} d A,
$$

with $k$ the Gaussian curvature of $\partial K$ at $p$, where $n$ is the unit vector normal to $T_{p} \partial K$, and where $d A$ is the Euclidean area.

To introduce the centroprojective area, we will consider a compact convex subset of the (real) $n$-dimensional projective space. Here the word "convex" means that each intersection with a projective line is connected. 
The definitions of the centroprojective seminorm and area are merely the same as the centroaffine ones, but one has to replace the Minkowski functional by a projectively invariant function.

Definition 4.4. Let $K \subset \mathbb{P}^{n}$ be a convex body and $o \in$ int $K$. The projective gauge function is

$$
G_{K}: \mathbb{P}^{n} \backslash\{o\} \rightarrow \mathbb{R} \cup\{\infty\}, \quad x \mapsto 2\left[q_{1}, o, x, q_{2}\right],
$$

where $q_{1}$ and $q_{2}$ are the two intersections of $\partial K$ with the line going through $o$ and $x$.

Since the order of $q_{1}$ and $q_{2}$ is not fixed, this function is multivalued (in fact double-valued). Identifying $\mathbb{R} \cup\{\infty\}$ with $\mathbb{P}^{1}$, this function is continuous.

If $p$ belongs to the boundary of $K$, then the two values of $G_{K}(p)$ are different, one of them being 2, the other being $\infty$. Hence there is some neighborhood $U$ of $p$ such that the restriction of $G_{K}$ to $U$ is the union of two continuous (in fact smooth) functions $G_{K}^{+}$and $G_{K}^{-}$on $U$, where $G_{K}^{+}(p)=2$ and $G_{K}^{-}(p)=\infty$.

Let $v$ be a tangent vector to $\partial K$ at a smooth point $p$. Since the restriction of $G_{K}^{+}$to $\partial K \cap U$ is constant, the derivative of $G_{K}^{+}$in the direction of $v$ vanishes. Therefore, the Hessian of the restriction of $G_{K}^{+}$to the tangent line is well defined.

Definition 4.5. The centroprojective seminorm of $v$ is

$$
\|v\|_{p}:=\sqrt{\operatorname{Hess}_{p} G_{K}^{+}(v, v)} .
$$

If we let $\omega_{p}$ be the induced volume form on $\partial K$, the centroprojective area of $K$ is $\mathscr{A}_{p}(K):=\int_{\partial K}\left|\omega_{p}\right|$.

Proposition 4.6. In a Euclidean space,

$$
\mathscr{A}_{p}(K)=\int_{\partial K} \frac{\sqrt{k}}{\langle n, p\rangle^{(n-1) / 2}}\left(\frac{2 a}{1+a}\right)^{(n-1) / 2} d A .
$$

In particular, the intrinsic definition of $\mathscr{A}_{p}$ agrees with the definition given in the introduction.

Proof. An easy computation shows that

$$
\left[q_{1}, o, x, q_{2}\right]=\frac{1+a\left(q_{2}\right)}{F(x)+a\left(q_{2}\right)} F(x) .
$$

Then, if $p$ is a smooth point of $\partial K$ and $v \in T_{p} \partial K$,

$$
\operatorname{Hess}_{p} G_{K}(v, v)=\frac{2 a(p)}{1+a(p)} \operatorname{Hess}_{p} F(v, v) .
$$


4.2. Properties of the centroprojective area. Both centroaffine and centroprojective areas vanish on polytopes, and hence they are not continuous with respect to the Hausdorff topology on (pointed) bounded convex bodies. Nevertheless, the centroaffine area is upper-semicontinuous [Lutwak 1996]. The same holds true for the centroprojective area as shown in the next theorem.

Theorem 4.7. The centroprojective area is finite, invariant under projective transformations, and upper-semicontinuous.

Proof. From the above intrinsic definition, it follows that $\mathscr{A}_{p}$ is invariant under projective transformations. Also, since the function $a$ on the boundary is bounded and positive, and since the centroaffine area is finite, it follows from Proposition 4.6 that the centroprojective area is also finite. It remains to show that it is uppersemicontinuous. Our proof is based on the fact that the centroaffine surface area $\mathscr{A}_{a}$ is semicontinuous [Lutwak 1996].

Let $K$ be a bounded convex body containing the origin in its interior, and let $\left(K_{i}\right)$ be a sequence of convex bodies with the same properties converging to $K$. Set

$$
\tau(p):=\left(\frac{2 a(p)}{1+a(p)}\right)^{(n-1) / 2} \text { for } p \in \partial K,
$$

which is a continuous function on $\partial K$.

For each $i$, if $a_{i}$ is the function corresponding to $K_{i}$ and $p_{i}$ is the radial projection of $p$ on $\partial K_{i}$, define $\tau_{i} \in C(\partial K)$ by

$$
\tau_{i}(p):=\left(\frac{2 a_{i}\left(p_{i}\right)}{1+a_{i}\left(p_{i}\right)}\right)^{(n-1) / 2} .
$$

Since $K_{i} \rightarrow K, \tau_{i}$ converges uniformly to $\tau$. Therefore $\left\|\tau_{i}-\tau\right\|_{\infty}<\epsilon$ for fixed $\epsilon>0$ and all sufficiently large $i$.

Take a triangulation of the sphere and let $\partial K=\bigcup_{j=1}^{m} \Delta_{j}$ be its radial projection. Define $\partial K_{i}=\bigcup_{j=1}^{m} \Delta_{i j}$ similarly.

Choosing this triangulation sufficiently thin, there exist $t_{1}, \ldots, t_{m} \in \mathbb{R}_{+}$such that $\left|\tau(p)-t_{j}\right|<\epsilon$ on $\Delta_{j}$. By the triangle inequality, $\left|\tau_{i}(p)-t_{j}\right|<2 \epsilon$ on $\Delta_{i j}$.

We define

$$
\mathscr{A}_{p}\left(K_{i}, \Delta_{i j}\right):=\int_{\Delta_{i j}} \frac{\sqrt{k(x)}}{\langle n(x), x\rangle^{(n-1) / 2}} \tau_{i} d \mathscr{H}^{n-1}(x) .
$$

Clearly, $\mathscr{A}_{p}\left(K_{i}\right)=\sum_{j=1}^{m} \mathscr{A}_{p}\left(K_{i}, \Delta_{i j}\right)$. We define $\mathscr{A}_{p}\left(K, \Delta_{j}\right), \mathscr{A}_{a}\left(K_{i}, \Delta_{i j}\right)$ and $\mathscr{A}_{a}\left(K, \Delta_{j}\right)$ similarly.

Fix $p_{j}$ in the interior of $\Delta_{j}$ and consider the convex hulls $\hat{\Delta}_{i}$ of $\Delta_{j} \cup\left\{-p_{j}\right\}$ and $\hat{\Delta}_{i j}$ of $\Delta_{i j} \cup-p_{j}$. The boundary of $\hat{\Delta}_{i j}$ is the union of $\Delta_{i j}$ and flat simplices; 
hence $\mathscr{A}_{a}\left(K_{i}, \Delta_{i j}\right)=\mathscr{A}_{a}\left(\hat{\Delta}_{i j}\right)$. By the semicontinuity of $\mathscr{A}_{a}$, we obtain

$$
\limsup _{i \rightarrow \infty} \mathscr{A}_{a}\left(K_{i}, \Delta_{i j}\right)=\limsup _{i \rightarrow \infty} \mathscr{A}_{a}\left(\hat{\Delta}_{i j}\right) \leq \mathscr{A}_{a}\left(\hat{\Delta}_{j}\right)=\mathscr{A}_{a}\left(K, \Delta_{j}\right) .
$$

It follows that

$$
\begin{aligned}
\limsup _{i \rightarrow \infty} \mathscr{A}_{p}\left(K_{i}\right) & =\limsup _{i \rightarrow \infty} \sum_{j=1}^{m} \mathscr{A}_{p}\left(K_{i}, \Delta_{i j}\right) \\
& \leq \limsup _{i \rightarrow \infty} \sum_{j=1}^{m} \mathscr{A}_{a}\left(K_{i}, \Delta_{i j}\right)\left(t_{j}+2 \epsilon\right) \leq \sum_{j=1}^{m} \mathscr{A}_{a}\left(K, \Delta_{j}\right)\left(t_{j}+2 \epsilon\right) .
\end{aligned}
$$

On the other hand,

$$
\mathscr{A}_{p}(K)=\sum_{j=1}^{m} \mathscr{A}_{p}\left(K, \Delta_{j}\right) \geq \sum_{j=1}^{m} \mathscr{A}_{a}\left(K, \Delta_{j}\right)\left(t_{j}-\epsilon\right),
$$

from which we deduce that $\lim \sup _{i \rightarrow \infty} \mathscr{A}_{p}\left(K_{i}\right) \leq \mathscr{A}_{p}(K)+3 \epsilon \mathscr{A}_{a}(K)$.

The centroaffine surface area has the following important properties:

- $\mathscr{A}_{a}$ is a valuation on the space of compact convex subsets of $V$ containing $o$ in the interior. This means that whenever $K, L, K \cup L$ are such bodies, then

$$
\mathscr{A}_{a}(K \cup L)=\mathscr{A}_{a}(K)+\mathscr{A}_{a}(L)-\mathscr{A}_{a}(K \cap L) .
$$

- $A_{a}$ is upper semicontinuous with respect to the Hausdorff topology.

- $\mathscr{A}_{a}$ is invariant under GL(V).

A recent theorem by M. Ludwig and M. Reitzner [2007] states that the vector space of functionals with these three properties is generated by the constant valuation and $\mathscr{A}_{a}$. The centroprojective surface area satisfies the last two conditions, but is not a valuation.

\section{Acknowledgments}

We wish to thank Bruno Colbois and Daniel Hug for interesting discussions and Franz Schuster for useful remarks on an earlier version of this paper.

\section{References}

[Alexandroff 1939] A. D. Alexandroff, "Almost everywhere existence of the second differential of a convex function and some properties of convex surfaces connected with it", Leningrad State Univ. Annals Math. Ser. 6 (1939), 3-35. MR 2,155a

[Álvarez Paiva and Fernandes 1998] J. C. Álvarez Paiva and E. Fernandes, "Crofton formulas in projective Finsler spaces”, Electron. Res. Announc. Amer. Math. Soc. 4 (1998), 91-100. MR 99j:53097 Zbl 0910.53044 
[Álvarez Paiva and Thompson 2004] J. C. Álvarez Paiva and A. C. Thompson, "Volumes on normed and Finsler spaces", pp. 1-48 in A sampler of Riemann-Finsler geometry, edited by D. Bao et al., Math. Sci. Res. Inst. Publ. 50, Cambridge Univ. Press, 2004. MR 2006c:53079 Zbl 1078.53072

[Bangert 1999] V. Bangert, "Convex hypersurfaces with bounded first mean curvature measure", Calc. Var. Partial Differential Equations 8:3 (1999), 259-278. MR 2000f:53043 Zbl 0960.53007

[Barvinok 2002] A. Barvinok, A course in convexity, Graduate Studies in Mathematics 54, American Mathematical Society, Providence, RI, 2002. MR 2003j:52001 Zbl 1014.52001

[Benoist 2004] Y. Benoist, "Convexes divisibles, I", pp. 339-374 in Algebraic groups and arithmetic, edited by S. G. Dani and G. Prasad, Tata Inst. Fund. Res., Mumbai, 2004. MR 2005h:37073 Zbl 1084.37026

[Benoist 2006] Y. Benoist, "Convexes hyperboliques et quasiisométries”, Geometriae Dedicata 122 (2006), 109-134. MR 2007k:20091 Zbl 1122.20020

[Benoist 2008] Y. Benoist, “A survey on divisible convex sets”, pp. 1-18 in Geometry, analysis and topology of discrete groups, edited by L. Ji et al., Adv. Lect. Math. (ALM) 6, Int. Press, Somerville, MA, 2008. MR 2464391 Zbl 1154.22016

[Bernig 2009] A. Bernig, "Hilbert geometry of polytopes", Arch. Math. (Basel) 92:4 (2009), 314324. MR 2010c:53106 Zbl 1171.53046

[Blaschke 1956] W. Blaschke, Kreis und Kugel, de Gruyter, Berlin, 1956. MR 17,1123d Zbl 0070.17501

[Borisenko and Olin 2008] A. A. Borisenko and E. A. Olin, "Asymptotic properties of Hilbert geometry", Zh. Mat. Fiz. Anal. Geom. 4:3 (2008), 327-345. MR 2444106 Zbl 1167.53066

[Bridson and Haefliger 1999] M. R. Bridson and A. Haefliger, Metric spaces of non-positive curvature, Grundlehren der Math. 319, Springer, Berlin, 1999. MR 2000k:53038 Zbl 0988.53001

[Colbois and Vernicos 2007] B. Colbois and C. Vernicos, "Les géométries de Hilbert sont à géométrie locale bornée”, Ann. Inst. Fourier (Grenoble) 57:4 (2007), 1359-1375. MR 2008k:53160 Zbl 1123.53022

[Colbois and Verovic 2004] B. Colbois and P. Verovic, "Hilbert geometry for strictly convex domains”, Geometriae Dedicata 105 (2004), 29-42. MR 2005e:53111 Zbl 1078.52002

[Colbois et al. 2008] B. Colbois, C. Vernicos, and P. Verovic, "Hilbert geometry for convex polygonal domains", preprint, 2008. arXiv 0804.1620

[Evans and Gariepy 1992] L. C. Evans and R. F. Gariepy, Measure theory and fine properties of functions, CRC Press, Boca Raton, FL, 1992. MR 93f:28001 Zbl 0804.28001

[Foertsch and Karlsson 2005] T. Foertsch and A. Karlsson, "Hilbert metrics and Minkowski norms", J. Geom. 83:1-2 (2005), 22-31. MR 2007e:51021 Zbl 1084.52008

[Gallot et al. 2004] S. Gallot, D. Hulin, and J. Lafontaine, Riemannian geometry, 3rd ed., Springer, Berlin, 2004. MR 2005e:53001 Zbl 1068.53001

[de la Harpe 1993] P. de la Harpe, “On Hilbert's metric for simplices”, pp. 97-119 in Geometric group theory (Sussex, 1991), vol. 1, edited by G. A. Niblo and M. A. Roller, London Math. Soc. Lecture Note Ser. 181, Cambridge Univ. Press, 1993. MR 94i:52006 Zbl 0832.52002

[Hilbert 1895] D. Hilbert, "Ueber die gerade Linie als kürzeste Verbindung zweier Punkte", Math. Ann. 46 (1895), 91-96. JFM 26.0540.02

[Hilbert 1999] D. Hilbert, Grundlagen der Geometrie, 14th ed., Teubner-Archiv zur Mathematik. Supplement 6, Teubner, Stuttgart, 1999. MR 2000j:01120 Zbl 0933.01031

[Hörmander 2007] L. Hörmander, Notions of convexity, Birkhäuser, Boston, MA, 2007. MR 2311920 Zbl 1108.32001 
[Hug 1998] D. Hug, "Absolute continuity for curvature measures of convex sets, I", Math. Nachr. 195 (1998), 139-158. MR 99j:52002 Zbl 0938.52003

[Hug 1999a] D. Hug, “Absolute continuity for curvature measures of convex sets, II", Math. Z. 232:3 (1999), 437-485. MR 2000m:52009 Zbl 0954.52006

[Hug 1999b] D. Hug, Measures, curvatures and currents in convex geometry, Habilitationsschrift, Universität Freiburg, 1999.

[Karlsson and Noskov 2002] A. Karlsson and G. A. Noskov, "The Hilbert metric and Gromov hyperbolicity”, Enseign. Math. (2) 48:1-2 (2002), 73-89. MR 2003f:53061 Zbl 1046.53026

[Kay 1967] D. C. Kay, “The ptolemaic inequality in Hilbert geometries”, Pacific J. Math. 21 (1967), 293-301. MR 35 \#4820 Zbl 0147.22406

[Kelly and Paige 1952] P. J. Kelly and L. J. Paige, "Symmetric perpendicularity in Hilbert geometries", Pacific J. Math. 2 (1952), 319-322. MR 14,308g Zbl 0048.13302

[Kelly and Straus 1958] P. Kelly and E. Straus, "Curvature in Hilbert geometries", Pacific J. Math. 8 (1958), 119-125. MR 20 \#2748 Zbl 0081.16401

[Kelly and Straus 1968] P. Kelly and E. G. Straus, "Curvature in Hilbert geometries, II”, Pacific J. Math. 25 (1968), 549-552. MR 38 \#613

[Koldobsky 2005] A. Koldobsky, Fourier analysis in convex geometry, Mathematical Surveys and Monographs 116, Amer. Math. Soc., Providence, RI, 2005. MR 2006a:42007 Zbl 1082.52002

[Laugwitz 1965] D. Laugwitz, Differentialgeometrie in Vektorräumen, unter besonderer Berücksichtigung der unendlichdimensionalen Räume, Vieweg, Braunschweig, 1965. MR 32 \#406

[Leichtweiß 1993] K. Leichtweiß, "Convexity and differential geometry”, pp. 1045-1080 in Handbook of convex geometry, vol. B, edited by P. M. Gruber and J. M. Wills, North-Holland, Amsterdam, 1993. MR 94j:52002 Zbl 0840.53038

[Leichtweiß 1998] K. Leichtweiß, Affine geometry of convex bodies, Barth, Heidelberg, 1998. MR 2000j:52005 Zbl 0899.52005

[Ludwig and Reitzner 2007] M. Ludwig and M. Reitzner, "A classification of SL $(n)$ invariant valuations", preprint, 2007. To appear in Ann. Math.

[Lutwak 1996] E. Lutwak, "The Brunn-Minkowski-Firey theory, II: Affine and geominimal surface areas”, Adv. Math. 118:2 (1996), 244-294. MR 97f:52014

[Mattila 1995] P. Mattila, Geometry of sets and measures in Euclidean spaces, Cambridge Studies in Advanced Mathematics 44, Cambridge Univ. Press, 1995. MR 96h:28006 Zbl 0819.28004

[Nasu 1961] Y. Nasu, “On Hilbert geometry”, Math. J. Okayama Univ. 10 (1961), 101-112. Zbl 0103.37901

[Schütt and Werner 1990] C. Schütt and E. Werner, "The convex floating body", Math. Scand. 66:2 (1990), 275-290. MR 91i:52005

[Shen 2001] Z. Shen, Lectures on Finsler geometry, World Scientific, Singapore, 2001. MR 2002f:53032 Zbl 0974.53002

[Socié-Méthou 2004] E. Socié-Méthou, "Behaviour of distance functions in Hilbert-Finsler geometry”, Differential Geom. Appl. 20:1 (2004), 1-10. MR 2004i:53112 Zbl 1055.53057

[Vernicos 2005] C. Vernicos, "Introduction aux géométries de Hilbert", pp. 145-168 in Actes de Séminaire de Théorie Spectrale et Géométrie, Sémin. Théor. Spectr. Géom. 23, Univ. Grenoble I, 2005. MR 2007m:51011 Zbl 1100.53031

[Vernicos 2008a] C. Vernicos, "Entropie volumique des géométries de Hilbert", preprint, 2008, Available at http://tinyurl.com/ydby7jt. To appear in Actes de Séminaire de Théorie Spectrale et Géométrie. 
[Vernicos 2008b] C. Vernicos, "Lipschitz characterisation of polytopal Hilbert geometries", preprint, 2008. arXiv 0812.1032

Received March 12, 2009. Revised September 24, 2009.

\title{
GAUTIER BERCK
}

DÉPARTEMENT DE MATHÉMATIQUES

CHEMIN DU MUSÉE 23

1700 FRIBOURG

SWITZERLAND

gautier.berck@unifr.ch

http://perso.unifr.ch/gautier.berck

\author{
ANDREAS BERNIG \\ INSTITUT FÜR MATHEMATIK \\ JOHANN WOLFGANG GOETHE-UNIVERSITÄT FRANKFURT \\ ROBERT-MAYER-STR. 10 \\ D-60054 FRANKFURT \\ GERMANY \\ bernig@math.uni-frankfurt.de \\ http://www.uni-frankfurt.de/fb/fb12/mathematik/an/bernig
}

CONSTANTIN VERnicos

DEPARTMENT OF MATHEMATICS

Logic House, South CAMPus

NATIONAL UNIVERSITY OF IRELAND, MAYNOOTH

Co. KILDARE

IRELAND

Constantin.Vernicos@maths.nuim.ie http://costia.free.fr/ 